\title{
LOS CAMBIOS CLIMÁTICOS GLOBALES Y EL DESAFÍO DE LA CIUDADANÍA PLANETARIA*
}

\author{
Fabio José Feldmann* y Rachel Biderman Furriela* ${ }^{*}$
}

Resumen: En este texto intentamos examinar la problemática del cambio climático cuyas consecuencias son cruciales para el provenir del planeta. Esta temática, legitimada y explicitada a través de distintas reuniones mundiales, se encuentra aún poco desarrollada en América Latina, a pesar de que sus consecuencias tendrán impactos decisivos en nuestra Región. La solución de los desafíos planteados por el cambio climático se expresa esencialmente en el ámbito de la ciudadanía, lo que supone la decisión de generar nuevas formas de diálogo, de circulación de la información, de educación y de democratización en la toma de decisiones.

Palabras clave: medio ambiente, ética ambiental, cambio climático, acuerdos internacionales.

\section{GLOBAL CLIMATIC CHANGES AND THE CHALLENGE OF PLANETARY CITIZENRY}

Abstract: In this paper we pretend to examine the issue of climatic changes and their crucial consequences for the future of the planet. This subject has been legitimized and made explicit through various world fora, but has undergone poor development in Latin America, although the serious and overriding impacts for the Region. The solution to challenges posed by climatic changes will fundamentally be expressed in the sphere of citizenry and that implies the creation of new forms of dialogue, of dissemination of information, of education, and a process of democratization in decision-making.

Keywords: environment, environmental ethics, climatic changes, international agreements.

\section{AS MUDANÇAS CLIMÁTICAS GLOBAIS E O DESAFIO DA CIDADANIA PLANETÁRIA}

Resumo: Neste texto pretendemos examinar a problemática das mudanças climáticas cujas conseqüências são cruciais para o futuro do planeta. Esta temática é legitima e é explicitada através de diferentes reuniões mundiais, porém é pouco desenvolvida na América Latina, apesar dos graves e decisivos impactos para a Região. A solução dos desafios apresentados pelas mudanças do clima se expressa fundamentalmente no âmbito da cidadania, o que supõe decisão de gerar novas formas de diálogo, circulação da informação, educação e democratização nas tomadas de decisões.

Palavras chaves: meio ambiente, ética ambiental, mudança climática, acordos internacionais.

- Traducido del Portugués por Paulina Lobos.

* Abogado. Secretario Ejecutivo del Fórun Brasilero de Mudanças Climáticas.

** Abogada, Master en Derecho Internacional (LL.M. - American University, Washington College of Law, EUA). Secretaria Ejecutiva del Instituto Pró-Sustentabilidade (IPSUS). 


\section{El fenómeno del cambio climático}

El siglo XX fue testigo de extraordinarios cambios, tanto en la sociedad como en el medio ambiente. Uno de los fenómenos actuales más preocupantes es la alteración del sistema climático global. La opinión pública y los gobernantes aumentaron su grado de percepción y preocupación respecto a este fenómeno principalmente debido a incidentes climáticos de relevancia tales como El Niño, inundaciones y otros desastres en distintas partes del mundo (Francia, España, Venezuela, Inglaterra, Australia, Canadá e India). Australia registró 1998 como el año más caluroso de su historia, y el Reino Unido sufrió en el año 2000 las peores tempestades registradas desde el siglo XVII. Esta preocupación está plasmada en los medios de comunicación. La revista Time publicó un reportaje, como noticia de tapa, sobre los "refugiados ambientales" en el 2000. Los cambios climáticos también fueron considerados por los dirigentes presentes en la Conferencia Económica de Davos, en febrero de 2000, como uno de los tres mayores problemas de la actualidad. Más recientemente, la posición de la Administración de los EEUU de rechazar el Protocolo de Kioto generó protestas en todo el mundo, movilizando a los medios de comunicación internacionales e impulsando a los gobernantes de Europa a asumir el liderazgo en las negociaciones internacionales sobre el clima. Los principales medios de comunicación publicaron noticias destacadas sobre las declaraciones del Presidente G. W. Bush y sus repercusiones. Un acontecimiento emblemático fue que, nuevamente la revista Time, en marzo del 2001, publicó como noticia de tapa el calentamiento global y sus implicaciones.

Las previsiones y registros de eventos climáticos extremos señalados anteriormente, no son producto de visiones catastróficas de los ambientalistas radicales. Este escenario fue identificado por los cientistas del Panel Intergubernamental sobre Cambios Climáticos que asesora a las Naciones Unidas desde 1988. El primer informe del IPCC, publicado en 1990, constató un aumento del $0,5^{\circ} \mathrm{C}$ en el promedio de la temperatura global, con relación al siglo anterior y alertó sobre la necesidad de tomar medidas severas para disminuir la emisión de gases causantes del efecto invernadero, como única manera de evitar el calentamiento global. Sobre la base de dichos estudios se inició una serie de negociaciones que culminaron en la Convención sobre el Clima, firmada durante la Conferencia de las Naciones Unidas sobre Medio Ambiente y Desarrollo, realizada en Río de Janeiro, en 1992. El tercer y último informe del IPCC, divulgado a principios del 2001, confirma los resultados del segundo informe de 1995, en el que se constata la relación entre el aumento de la temperatura y las actividades humanas y alerta sobre la necesidad urgente de tomar medidas para la reducción de emisiones de gases causantes del efecto invernadero.

Es importante aclarar que el efecto invernadero es un fenómeno natural, necesario a la mantención de la vida en el Planeta. Los gases del efecto invernadero actúan como una frazada alrededor de la Tierra, sin la cual ésta sería más fría. Se transformó en un problema debido al gran volumen de emisiones de gases desde el acontecimiento de la Revolución Industrial. Las actividades del hombre están aumentando el poder del efecto invernadero, más específicamente a través de:

- $\quad$ quema de carbón, petróleo y gas natural por la industria y sistemas de transportes, que causan importantes emisiones de gas carbónico;

- $\quad$ destrucción de los bosques y diferentes formaciones de vegetación y cambios en el uso del suelo, porque el carbono almacenado en la vegetación y en el suelo se escapa hacia la atmósfera;

- $\quad$ crianza de ganado y cultivo del arroz, actividades que emiten metano, óxido nitroso y otros gases de efecto invernadero,

- degradación de residuos en espacios sanitarios o basurales que emiten metano.

Todos los países son fuentes de emisiones de $\mathrm{CO}_{2}$ pero la magnitud y la diversidad de las fuentes varían de acuerdo con el país y la región. Gran parte del carbono emitido históricamente por la quema de combustibles fósiles se origina en los países industrializados. Las emisiones en los países 
en desarrollo, entre tanto, ha aumentado bastante $\mathrm{y}$, con la confirmación de dicha tendencia, en la segunda década de este siglo más de la mitad de las emisiones tendrán su origen en dichos países. La deforestación y las alteraciones del uso del suelo son las principales fuentes de emisión de $\mathrm{CO}_{2}$ en países en desarrollo. Si las emisiones siguen creciendo al ritmo actual, es casi seguro que los niveles de dióxido de carbono en la atmósfera van a ser, en el siglo 21, dos veces mayores que las concentraciones del período pre-industrial.

Los cientistas del IPCC identifican algunos posibles impactos como consecuencia del aumento del promedio de la temperatura en el planeta. Algunos de estos efectos son:

\section{$\bullet$}

los regímes regionales de lluvia y padrones de viento pueden cambiar: algunas regiones del mundo corren el riesgo de volverse más lluviosas y otras, más secas;

- $\quad$ las zonas climáticas y agrícolas pueden migrar;

- $\quad$ el deshielo y la dilatación térmica de los océanos pueden causar el aumento del nivel de los océanos, amenazando zonas de la costa de baja altitud y pequeñas islas;

- las enfermedades propagadas por vectores asociados al cambio de temperatura, como el dengue y la malaria, por ejemplo, podrían potencializar su incidencia;

- $\quad$ los impactos sobre los recursos hídricos van a aumentar la escasez de agua para sus múltiples usos;

- los países en desarrollo o menos desarrollados no tendrán recursos suficientes para prepararse contra los impactos o minimizar sus efectos, lo que generará graves consecuencias con impactos sociales y económicos;

- las regiones áridas se podrían transformar en desiertos y las regiones secas se podrían volver aún más secas,

- reducción del potencial de la producción alimentaria, generando mayores problemas de hambre y miseria.

La búsqueda de soluciones para dicho problema universal ha sido objeto de discusiones, conferencias y acuerdos internacionales desde el final de la década de los 80. Las Naciones Unidas establecieron una agenda internacional de compromisos con el objeto de solucionar los problemas que afectan a la sociedad, la economía y el medio ambiente. Dos son los principales tratados que reglamentan las iniciativas para contener los efectos del calentamiento global: la Convención Marco de las Naciones Unidas sobre Cambio del Clima y el Protocolo de Kioto, anexo a esta convención. Además de estos dos documentos principales, la Agenda 21, tratado internacional adoptado en 1992 por las Naciones Unidas, que estableció un importante plan de acción para promover el desarrollo viable en el planeta. Trata también de las variaciones climáticas en su capítulo 9 sobre Protección de la Atmósfera.

\section{Los tratados internacionales}

\section{La Convención sobre el Cambio del Clima}

La Convención Marco de las Naciones Unidas sobre el Cambio de Clima estableció un compromiso general de reducción de la emisión de gases de efecto invernadero. Es un tratado genérico, que solamente contiene algunas exigencias específicas. La Convención se adoptó el 9 de mayo de 1992 en la sede de las Naciones Unidas en Nueva York y fue abierta para la firma en 1992 en la Cumbre de la Tierra en Río de Janeiro. Fue firmada en dicho encuentro por Jefes de Estado y otras autoridades de 154 países y la Comunidad Europea. Entró en vigencia el 21 de marzo de 1994. Brasil firmó la Convención durante la Cumbre de la Tierra (Río 92), el 04 de junio de 1992 y se ractificó el 28 de febrero de 1994.

La Convención estableció en su Preámbulo una serie de premisas que deben orientar las acciones de los Estados en sus acciones de lucha contra los cambios climáticos. Entre los preceptos básicos se destaca que el cambio del clima y sus efectos son una preocupación común de la humanidad. Se reconoce que las actividades humanas aumentan las concentraciones de gases de efecto invernadero, lo que provoca un calentamiento adicional de la superficie y de la atmósfera de la Tierra, que afecta los ecosistemas naturales y la humanidad. Además, 
los países signatarios entienden que la mayor parte de las emisiones históricas y actuales tienen su origen en los países desarrollados y que la naturaleza global del cambio de clima requiere la mayor cooperación posible de todos los países que tomen en consideración "sus responsabilidades comunes más diferenciadas" y sus respectivas capacidades y condiciones sociales y económicas. Entre los fundamentos de La Convención también se establece que las actividades de un Estado no deben causar daño al medio ambiente de otros Estados y que los Estados deben elaborar una legislación ambiental eficaz para combatir el problema. Los países reconocen la urgencia de adoptar medidas inmediatas por parte de los países desarrollados, considerándose sus contribuciones relativas en el aumento del efecto invernadero. Reconocen también que los países de baja altitud, insulares, con zonas de costa de baja altitud, regiones áridas y semiáridas, sujetas a inundaciones, sequía y desertificación, y países en desarrollo con ecosistemas montañosos frágiles son particularmente vulnerables a los efectos negativos del cambio del clima. Otra premisa es que la lucha contra el cambio climático debe garantizar el desarrollo social y económico considerando las prioridades de los países en desarrollo y que el consumo de energía de los países en desarrollo debe aumentar para que alcancen el desarrollo económico. Las buenas intenciones del Preámbulo incluyen también la previsión de que será necesario proteger el sistema climático para las generaciones presentes y futuras.

Las premisas arriba enunciadas fundamentan el objetivo central de La Convención, previsto en su artículo $2^{\circ}$ :

"Alcanzar la estabilización de las concentraciones de gases de efecto invernadero en la atmósfera a un nivel que impida una interferencia antrópica peligrosa en el sistema climático. Este nivel deberá alcanzarse en un plazo suficiente que permita que los ecosistemas se adapten naturalmente al cambio del clima, que asegure que la producción de alimentos no sea amenazada y que permita que el desarrollo económico siga

\section{de forma sostenible."}

Se trata de una convención que es un hito en el Derecho Internacional y en las Relaciones Internacionales, porque es la primera vez en la historia en que países de todo el mundo reconocieron que las actividades humanas están causando serios impactos sobre el medio ambiente y pueden amenazar la continuidad de la vida en el planeta e imponen medidas concretas para contener el problema. Lo inédito de la solución propuesta ha asustado a los gobernantes y a los tomadores de decisión de los grandes grupos económicos, puesto que la reducción de emisión de gases de efecto invernadero implicará la transformación de los actuales padrones de consumo y producción y de su base energética, dependiente del uso de combustibles fósiles.

\section{El Protocolo de Kioto}

En diciembre de 1997, la Conferencia de las Partes de la Convención sobre Cambio del Clima aprobó en Kioto, Japón, un tratado internacional que se conoció como "Protocolo de Kioto". El Protocolo establece que los países desarrollados estarán obligados a reducir sus emisiones colectivas de seis gases efecto invernadero en por lo menos 5\%, comparados con los niveles de 1990, para el período entre los años 2008-2012. Con el pasar de los años y con la aprobación de acuerdos recientes en Bonn (COP 6 - julio 2001) y Marrakech (COP 7 - noviembre 2001) y con el rechazo de los Estados Unidos en ratificar el Protocolo, el compromiso de reducción de emisiones acordado en Kioto se debilitó. Después de los acuerdos de Bonn y de Marrakech algunos cientistas y ambientalistas han afirmado que las concesiones hechas en las negociaciones habrían implicado la reducción de este porcentaje mínimo al 1,5 o al $2 \%$, lo que es insuficiente para resolver el problema.

Según El Protocolo, los países tendrán cierta flexibilidad en el establecimiento de medidas para la reducción de emisiones y en el cálculo de estas reducciones. El Protocolo estableció algunos mecanismos de flexibilización para la implementación de las 
obligaciones para los países con metas de reducción de emisiones. Estos mecanismos son:

- $\quad$ Ejecución Conjunta (o Joint Implementation - JI), a ser realizado apenas entre países industrializados del Anexo 1

- $\quad$ Comercio de Emisiones (o Emissions Trading - ET), a ser realizado entre países del Anexo 1

- Mecanismo de Desarrollo Limpio (o Clean Development Mechanism - CDM), entre países industrializados (Anexo 1) y países en desarrollo.

El de mayor interés para América Latina es el Mecanismo de Desarrollo Limpio (MDL), único que se aplica a los países en desarrollo. Permite que países industrializados inviertan en proyectos de reducción de emisiones o plantas de tratamiento en países en desarrollo, disminuyendo parte de sus obligaciones en la reducción de emisiones. El Mecanismo aún no está implementado oficialmente, ya que sus reglas todavía no han sido completamente definidas. La expectativa de entrada en vigencia del mecanismo será a partir de 2002-2003, si el Protocolo es ratificado por un número suficiente de países según las reglas de éste. En realidad, el llamado "mercado de carbono" ya se encuentra en test, operando a partir de proyectos modelo de reducción de emisiones o de plantas de tratamiento, con la expectativa de reconocimiento y acreditación futura en el ámbito de las reglas del MDL.

El Protocolo de Kioto fue abierto para la ratificación el 16 de marzo de 1998. Entrará en vigencia 90 días después de su ratificación por al menos 55 partes de la Convención, incluyendo a los países desarrollados que contabilizarán por lo menos $55 \%$ de las emisiones totales de dióxido de carbono en 1990. Se espera que los países industrializados cumplan sus promesas hechas en Bonn y Marrakech (2001) y ratifiquen el Protocolo a tiempo para que entre en vigencia en el momento de la realización de la Conferencia de las Naciones Unidas sobre el Desarrollo Sustentable ${ }^{i}$, en Johanesburgo, África del Sur, donde se celebrarán los diez años de la
Convención sobre Cambio del Clima.

\section{Los desafíos}

El cambio climático global evidencia las desigualdades económicas y sociales que existen en el mundo. Potencializa dichas diferencias e inequidades. Los tratados del clima reconocen los diferentes niveles de responsabilidades y atribuyen a los países que más poluen (países industrializados) la mayor cuota de responsabilidades para la resolución del problema, puesto que históricamente ya consumieron y emitieron más gases que los países en desarrollo, usufructando, como consecuencia de estas emisiones, de los beneficios económicos, sociales y políticos. Entre algunas cifras que provocan susto, se encuentra la estimación de que un solo ciudadano norteamericano produce gases efecto invernadero equivalente al que producen 19 hindúes, 30 paquistanís o 269 nepaleses, ya que sus padrones de consumo implican gran consumo de combustibles fósiles y padrones del uso del suelo que generan grandes emisiones de gases efecto invernadero.

Este debate es aún incipiente en América Latina y se vuelve evidente la necesidad de que se entable la discusión respecto al tema con el público en general. Esta discusión no podrá prescindir de un enorme esfuerzo de traducción de esta compleja cuestión para que se incien en el tema, a través de proyectos de educación ambiental. De acuerdo con el tema, ésta deberá tener un enfoque transversal, involucrando todos los grandes currículos y todos los niveles de enseñanza. El público involucrado es vasto, puesto que incluye desde estudiantes hasta profesionales de los medios de comunicación, empresarios, financistas, gobernantes y ciudadanos comunes. Es un enorme desafío que se plantea a los organismos de gobierno, instituiciones de enseñanza, prensa y ONGs, que siempre estuvieron delante del movimiento

\footnotetext{
Esta será la tercera gran conferencia mundial sobre el tema ambiental. Su enfoque será sobre el desarrollo sustentable. Las dos anteriores ocurrieron en 1972 en Estocolmo, y en 1992, en Río de Janeiro.
} 
de concientización en relación con los temas ambientales.

Además de esta sensibilización, es fundamental que la sociedad se capacite técnicamente para que pueda enfrentar los impactos de los cambios climáticos. No es suficiente conocer el problema. Es necesario enfrentarlo. Enormes esfuerzos y grandes recursos se hacen necesarios en los países en desarrollo para adecuar sus parques industriales, cambiar formas de producción agrícola, preparar los gobiernos y las sociedades para enfrentar los efectos adversos de los cambios climáticos. De nada sirve poner todas las esperanzas en los mecanismos de mercado. Estos son importantes pero, ya quedó probado, son insuficientes e incapaces de resolver problemas ambientales y sociales, menos aún a nivel del planeta. La capacitación para combatir el problema debe ser profesional, depende de una gran inversión de recursos, que hasta hoy es mínima en América Latina. Será necesario que se formen más educadores, más cientistas ambientales, médicos, economistas, técnicos en salud, o sea, profesionales de todos los sectores para enfrentar las implicaciones del fenómeno. Inclusive la defensa civil de los países tendrá que capacitarse para manejar los impactos del aumento del nivel del mar, o las inundaciones devastadoras.

Será también necesario que se amplíen las bases de la democracia ambiental. Las decisiones que deben tomarse para la contención del fenómeno, tienen implicaciones económicas y sociales serias. Y la sociedad debe tener acceso a los mecanismos y espacios de decisión sobre el tema y, especialmente, acceso a la información pertinente para tomar las decisiones en forma consciente y consecuente. Es necesario que sean creados foros de concientización, discusión y capacitación, abriendo las instancias tomadoras de decisión a la consulta pública, capacitando a los ciudadanos para que participen en la definición de los rumbos de la gestión del tema público, asumiendo así, compromisos y riesgos.

Estamos frente a un problema cuya solución implica transformaciones reales del actual paradigma de desarrollo. Depende de la formación de una ciudadanía planetaria, de ciudadanos preocupados y comprometidos con el futuro de la vida en la Tierra. Esto supone la alteración de la matriz energética del planeta, en donde predomina el uso de combustibles fósiles. Será necesario "descarbonizar" las actividades humanas para que se pueda "descarbonizar" la atmósfera, evitando alteraciones climáticas peligrosas para la presencia humana en la Tierra y alteraciones relevantes de hábitats y otras formas de vida.

Este cambio de la matriz energética cambiará padrones de consumo y producción. Se hará necesario producir de manera más eficiente, no solamente bajo el punto de vista económico, sino también ambiental y energético. Será indispensable consumir de manera más eficaz, menos predatoria. El consumidor-ciudadano consciente será el actor de este nuevo paradigma. El consumidor consciente es el agente capaz de exigir del sector productivo una producción más limpia, más respetuosa de la vida y del ambiente.

El cambio de estilos de vida también se vuelve indispensable. Aquí se encuentran las mayores resistencias al proceso de transformación. Algunos países desarrollados alegan que no será necesario que sus ciudadanos, ya acostumbrados a un alto estándar de vida, dejen de vivir de manera tan cómoda. Pretextan que nuevos descubrimientos tecnológicos harán posible vivir tan bien como viven hoy y promover el equilibrio climático. Sólo el tiempo probará si de hecho es verdad. Pero, definitivamente, es preferible prevenir antes que curar. El hecho es que los cambios deben ser rápidos. Nos queda menos de un siglo para abandonar los combustibles fósiles, la producción industrial altamente poluyente, las acciones predatorias sobre los bosques y el manejo predatorio del suelo, a fin de garantizar la viabilidad de todas las formas de vida en la Tierra. Debemos aprender de lo que quedó demostrado en la última década, a partir de la Conferencia de Río de 1992: el desarrollo se debe controlar a través de criterios de sustentabilidad, respetando las exigencias de conservación ambiental, equidad, $\mathrm{y}$, las futuras generaciones, sometiéndose a procesos de participación y negociación social. 